\title{
A novel configuration of THz photonic transmitter
}

\author{
I. Moumane ${ }^{1}$, J. Zbitou ${ }^{2}$, M. Latrach ${ }^{3}$, A. Errkik ${ }^{4}$, O.Chakkor ${ }^{5}$ \\ ${ }^{1,2,4}$ Laboratory of Mechanics, Energetics, Electronics and Telecommunications LMEET, \\ FSTS Hassan 1St University of Settat, Morocco \\ ${ }^{3}$ Microwave group ESEO, Angers, France \\ ${ }^{5}$ ENSA of Tetuan, Morocco
}

\begin{tabular}{l}
\hline \hline Article Info \\
\hline Article history: \\
Received Sep 4, 2018 \\
Revised Nov 19, 2018 \\
Accepted Nov 30, 2018 \\
\hline
\end{tabular}

\section{Keywords:}

CW THz photonic transmitters

GaAs substrate

Low-pass filter

MSM-TPWD

\begin{abstract}
In this paper we present a new design of a photonic transmitter based on multi-layers substrate $\mathrm{GaAs}$ and using for generation of $\mathrm{THz}$ waves. The proposed circuit is composed from a photodetector (PD) "which converts the light to the electrical signal", THz Antenna, low-pass filter and DC probe. Firstly we have optimized the THz antenna using an EM solver which is Momentum integrated in ADS “Advanced Design System". Then we have optimized the low pass filter which plays the role of inductance that is an RF choke permiting to separate the RF signal from the DC one. Finally, we have associated the different components, add the DC probe and simulate the whole proposed system. The dimensions of the final circuit are $400.317 \times$ $167.16 \mu \mathrm{m}^{2}$. The obtain results permit to validate the final circuit at $\mathrm{THz}$ and make it suitable for $\mathrm{THz}$ applications.
\end{abstract}

Copyright $(0) 2019$ Institute of Advanced Engineering and Science. All rights reserved.

\section{Corresponding Author:}

I. Moumane,

Laboratory of Mechanics, Energetics, Electronics and Telecommunications LMEET,

FSTS Hassan 1St University of Settat, Morocco.

Email: moumaneibti@gmail.com

\section{INTRODUCTION}

Recently we remark the fast growing of the $\mathrm{THz}$ applications due to a lot of facturies related to the development of the modern Femtosecond Lasers and High-Speed Photodetectors. Many researchers in different domains such as biomedical imaging, spectroscopy, Security and telecommunications are focusing now theirs searchers on $\mathrm{THz}$ waves which presented several advantages based on interactivity with the material where it spreads and fast absorption by the atmosphere [1].

Before the difficulties of the $\mathrm{THz}$ domain reside in the generation and detection. But the situation are changed now, for the generation of the $\mathrm{THz}$ waves as an exemple many methods are proposed but the most used is the one that relays on the coplanar waveguide (CPW) photonic transmitters [2]. The CPW technology offers in fact several advantages due to its features, like low radiation, low dispersion, easy of shunts and series connections [3].

In this paper we present a compact photonic transmitter composed from a photodetector associated to the THz antenna, low pass filter and DC probe Bias. The following sections will study and discuss the design of the different components.

\section{PHOTONIC TRANSMITTER SYSTEM}

\subsection{Photodetector "PD"}

Photodetectors are semiconductor devices that can detect optical signals through electronic processes. The extension of wavelength of coherent and incoherent light sources into the far-infrared region on one hand and the ultraviolet region on the other has increased the need for high-speed, sensitive photodetectors. The operation of a general photodetector includes basically three processes: 
(1) carrier generation by incident light, (2) carrier transport andor multiplication by current-gain mechanism if present, and (3) extraction of carriers as terminal current to provide the output signal [4].

There exist many types of photodetectors. They are divided into two classes; thermal detectors and photon detectors. Thermal detectors detect light by sensing the temperature rise when the light energy is absorbed at their dark surface. These are more suitable for far-infrared wavelengths. Technically they are more like thermal sensors and will be discussed to a larger extent in the next chapter. The photon detec- tors are based on the quantum photoelectric effect: a photon excites a carrier which contributes to the photocurrent [4].

The photoelectric effect is based on the photon energy $\mathrm{h} v$, the wavelength of interest is related to energy transition $\mathrm{AE}$ in the device operation, with the obvious but important relationship [4].

$$
\lambda[\mu \mathrm{m}]=\frac{\mathrm{hc}}{\Delta \mathrm{E}}=\frac{1.24}{\Delta E(\mathrm{eV})}
$$

Where is $\lambda$ the wavelength, $\mathrm{c}$ is the speed of light, and $\Delta \mathrm{E}$ is the transition of energy levels.

A PD has different proprieties such as:

Sensitivity: The ability of the photodiode to transform light absorbed into an electrical current in other term the number of charge carrier pairs generated per incident photon [5].

$$
\eta_{\text {ext }}=\frac{I_{\mathrm{Pd}}}{\mathrm{q}} \cdot \frac{\mathrm{h} v}{\mathrm{P}_{\mathrm{opt}}}
$$

Responsivity: Where Ipd is the photogenerated current by the absorption of the optical input power Popt at a frequency $v$ mentied in (2). A common figure of merit is the external responsivity R, defined as the ratio of photocurrent to the input optical power [5]:

$$
\mathrm{R}=\frac{I_{\mathrm{Pd}}}{\mathrm{P}_{\mathrm{opt}}}=\frac{\eta_{\mathrm{ext}} \lambda[\mu \mathrm{m}]}{1.24} A / W
$$

Where $\mathrm{R}$ depends on the state of polarization of the incoming light, the definition of the polarization dependent loss (PDL) is useful [5]:

$$
\mathrm{PDL}=10 \cdot \log \left(\frac{R_{\max }}{R_{\min }}\right) d B
$$

With Rmax and Rmin are the maximum and minimum responsivities for all states of polarization.

In order to evaluate the high-speed photodiode, the electrical $3 \mathrm{~dB}$ bandwidth is used, It specifies the frequency range from $\mathrm{DC}$ to the cut-off frequency $\mathrm{f}_{3 \mathrm{~dB}}$. The bandwidth of a lumped element photodiode, at which the device length is much shorter than the electrical signal wavelength, might be limited mainly by the RC-time constant and the carrier drift times in the depletion region. These bandwidth constraints lead to a $3 \mathrm{~dB}$ bandwidth which can be approximated by [5]:

$$
\mathrm{f}_{3 \mathrm{~dB}} \approx \sqrt{\frac{1}{\frac{1}{\mathrm{f}_{\mathrm{RC}}^{2}+\frac{1}{\mathrm{f}_{\mathrm{t}}^{2}}}}}
$$

With the RC limited bandwidth [5].

$$
\mathrm{f}_{\mathrm{RC}}=\frac{1}{2 \pi \mathrm{R}_{\text {tot }} \mathrm{C}_{\mathrm{pd}}}
$$

And the carrier transit time limited bandwidth [5].

$$
\mathrm{f}_{\mathrm{t}}=\frac{3.5 \bar{\vartheta}}{2 \pi \mathrm{d}_{\mathrm{abs}}}
$$

$\mathrm{R}_{\text {tot }}$ stands for the photodiodes's total resistance including series and load resistances [5].

$\mathrm{C}_{\mathrm{pd}}$ is the junction capacitance [5].

$\bar{\vartheta}=\sqrt[4]{2\left(1 / v_{\mathrm{e}}^{4}+1 / \mathrm{v}_{\mathrm{h}}^{4}\right)^{-1}}$ is the averaged carrier drift velocity with the electron and hole velocities $V_{\mathrm{e}}$ and $V_{h}[5]$. 
$\mathrm{d}_{\mathrm{abs}}$ : The absorber thickness [5].

In this work we have chosen the Metal Semiconductor-Metal Traveling wave Photodetector (MSMTPD) by reason of its high power-bandwidth and coplanar-waveguide fed slot owing to its easy connection with planar devices [6]. The PD based on GaAs substrate which characterized by a succession of layers as mentioned in the Figure 1 [7]:

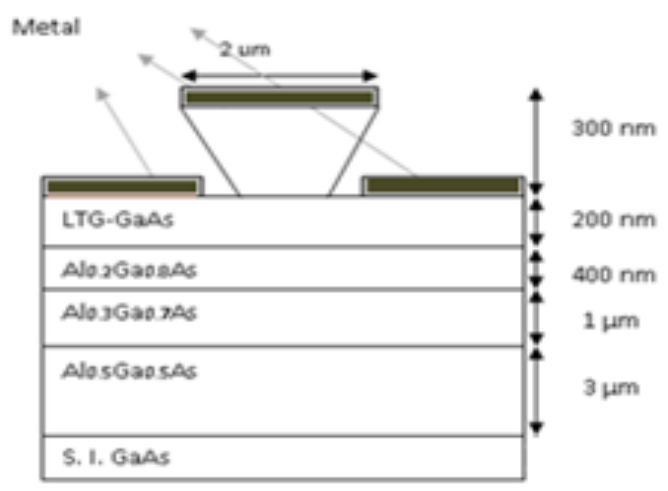

Figure 1. Structure of the photodetector based on GaAs substrate [7]

\subsection{THz Wideband Antenna}

The integration of the antenna into the $\mathrm{CW}$ photonic transmitter circuit is mandatory. It's role focuses on the transmition of the RF signal providing from the PD.

In the Figure in below we present the design of $\mathrm{THz}$ Antenna based on the multilayer substrate presented in Figure 1. The final dimensions are obtained after many series of optimization using different methods integrated in ADS as Random and gradient techniques. The bowtie antenna structure as shown in Figure 2 and final optimized parameters in Table 1.

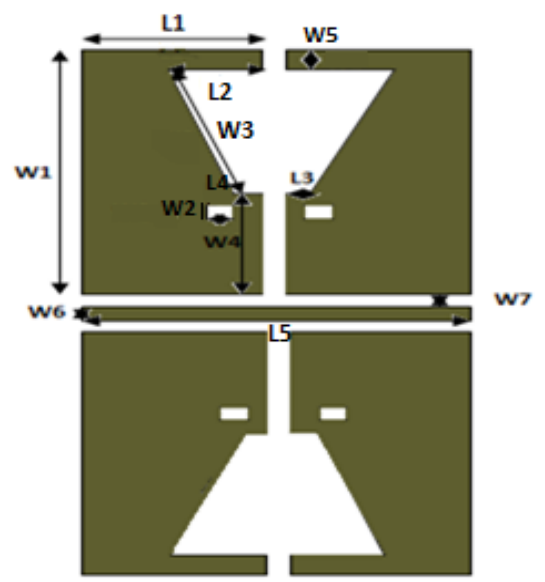

Table 1. The Final Optimized Parameters of the Proposed CPW THz Antenna

\begin{tabular}{cc}
\hline Parameter & Value $(\mu \mathrm{m})$ \\
\hline L1 & 55.09 \\
L2 & 26.39 \\
L3 & 6.93 \\
L4 & 8.24 \\
L5 & 116.77 \\
W1 & 79.17 \\
W2 & 5 \\
W 3 & 48.10 \\
W4 & 29.35 \\
W5 & 6.27 \\
W6 & 4 \\
W7 & 5 \\
\hline
\end{tabular}

Figure 2. The bowtie antenna structure

Figure 3 presents the reflection coefficient S11 of the validated antenna with a good matching impedance below $-10 \mathrm{~dB}$ in the bandwidth between $1 \mathrm{THz}$ and $1.28 \mathrm{THz}$. 


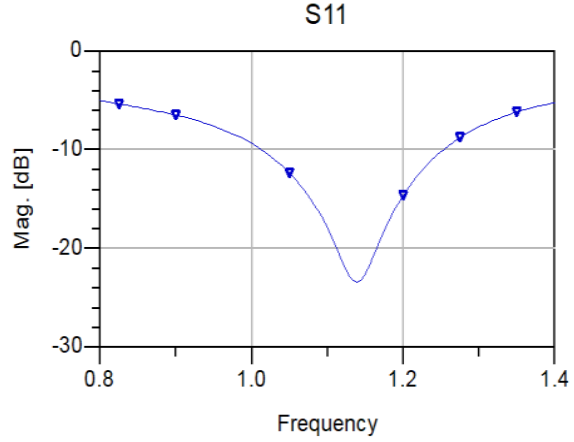

Figure 3. Reflection coefficient versus frequency

To have an idea about the behavior of the antenna we have launched a 3D simulation at $1.14 \mathrm{THz}$ in ADS, we obtain the radiation diagram which shows that the antenna is bidirectional. The radiation pattern at $1.14 \mathrm{THz}$ as shown in Figure 4.

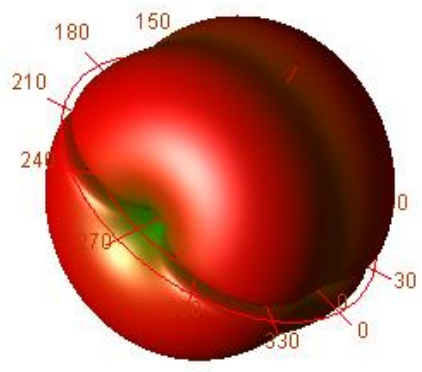

Figure 4. The radiation pattern at $1.14 \mathrm{THz}$

We have done a comparison between the proposed antenna and another $\mathrm{THz}$ antennas validated in literature to give an idea about the performances of the final validated $\mathrm{THz}$ antenna.The Table 2 presents the difference in term of (dimensions, frequency bandwidth) between the proposed antenna and others structures:

Table 2. Comparison of the Antenna structures

\begin{tabular}{lcc}
\hline \multicolumn{1}{c}{ Antenna Structure } & Length & Frequency Bandwidth \\
\hline Proposed Antenna & $117 \mu \mathrm{m}$ & {$[1 \mathrm{THz}, 1.28 \mathrm{THz}]$} \\
Antenna[8] & $330 \mu \mathrm{m}$ & {$[1 \mathrm{THz}, 1.25 \mathrm{THz}]$} \\
Antenna [9] & $200 \mu \mathrm{m}$ & Narrow band at $645 \mathrm{Ghz}$ \\
Antenna [10] & $1040 \mu \mathrm{m}$ & Narrow bandat $850 \mathrm{Ghz}$ \\
\hline
\end{tabular}

As shown in this table, the proposed antenna presents good performances in term of bandwidth and length.

\subsection{Low-Pass Filter "LPF"}

After the optimization of $\mathrm{THz}$ Antenna we pass to the second step the validation of the LPF [11], which is responsible of in blocking the RF signal providing from PD and transmitting via antenna to reach the DC probe Bias.

In this study we have chosen a a steped impedance LPF structure presenting in the study [12] with the aim to increase the rejection band. The proposed LPF composed from three unit cells as shown in Figure 5 and dimensions are presented in Table 3 . 


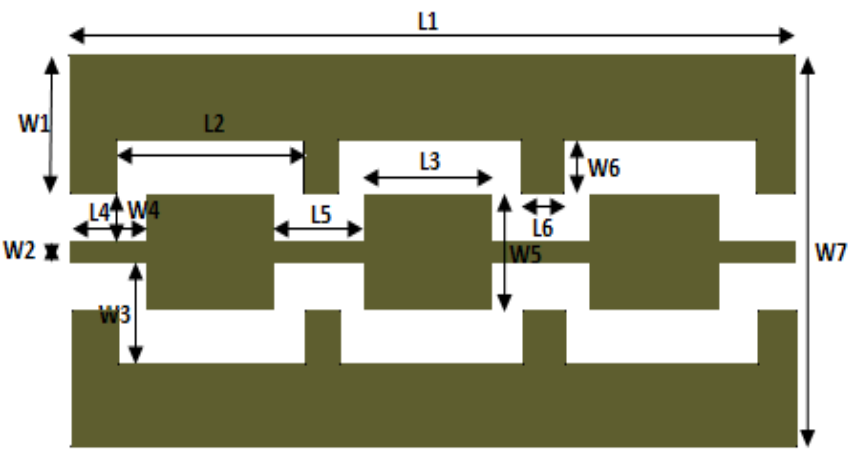

Figure 5. The layout of the periodic LPF THz structure
Table 3. Values of the LPF Parameters

\begin{tabular}{cc}
\hline Parameter & Value $(\mu \mathrm{m})$ \\
\hline L1 & 171 \\
L2 & 45.78 \\
L3 & 32.74 \\
L4 & 16.97 \\
L5 & 19.40 \\
L6 & 6.67 \\
W1 & 37.29 \\
W2 & 7 \\
W 3 & 19.40 \\
W4 & 10.91 \\
W5 & 32.44 \\
W6 & 9.09 \\
W7 & 97.62 \\
\hline
\end{tabular}

The Figure 6 presents a good low insertion loss, with a cutoff frequency of $0.88 \mathrm{THz}$ and a wide rejection band until 1.1 THz, which makes the proposed "LPF" suitable for THz applications.

To form an idea about the function of the proposed "LPF" structure we have launched a 3D simulation in $\mathrm{ADS}$ at $0.75 \mathrm{THz}$ in the frequency passband and at $1 \mathrm{THz}$ in the rejection frequency band. As a result, we can see from Figure 7 (a) that we have a flow of energy from port 1 to port 2 and for Figure 9 (b) the energy is stoped to reach the port 2.

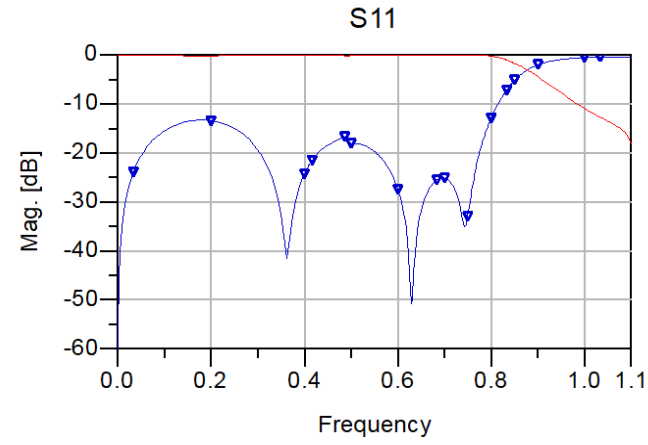

Figure 6. Simulation S-Parameters results versus frequency

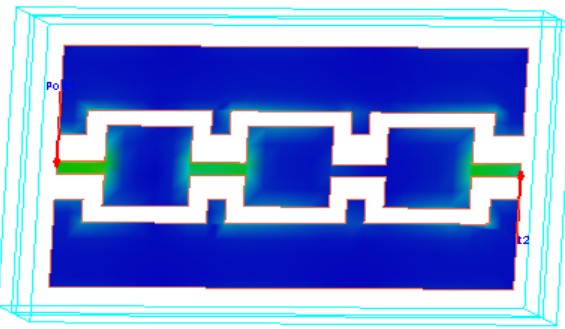

(a)

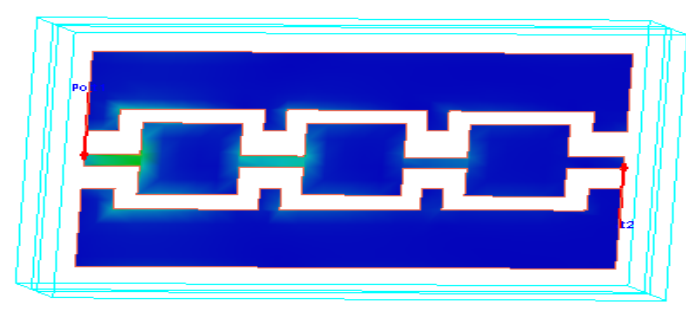

(b)

Figure 7. The current density @ $0.75 \mathrm{THz}$ (a) THz and (b) $1 \mathrm{THz}$

\subsection{CW Photonic Transmitter Simulation}

After the validation of the THz antenna and the LPF, we have connected the different components to the photodetector MSM-TWPD and added the DC probe responsible for the Bias of the PD to obtain the final circuit CW photonic transmitter presented in Figure 8 and values of DC probe parameters in Table 4. 


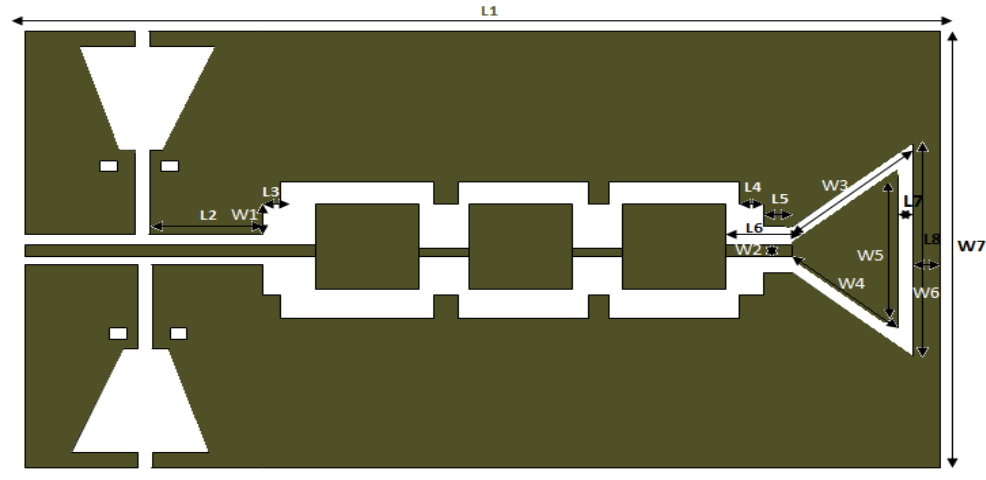

Figure 8. The proposed $\mathrm{CW}$ photonic transmitter
Table 4. Values of DC Probe

\begin{tabular}{cc}
\multicolumn{2}{c}{ Parameters } \\
\hline Parameter & Value $(\mu \mathrm{m})$ \\
\hline L1 & 400 \\
L2 & 54.75 \\
L3 & 9.42 \\
L4 & 10.15 \\
L5 & 19.58 \\
L6 & 35.17 \\
L7 & 6.16 \\
L8 & 7.61 \\
W1 & 6.52 \\
W2 & 5 \\
W 3 & 98.06 \\
W4 & 89.92 \\
W5 & 96.46 \\
W6 & 118.21 \\
W7 & 167.52 \\
\hline
\end{tabular}

The Figure 9 presents the simulation result of the $\mathrm{CW} \mathrm{THz}$ photonic transmitter which permits to validate this circuit between $1.26 \mathrm{THz}$ and $1.32 \mathrm{THz}$.

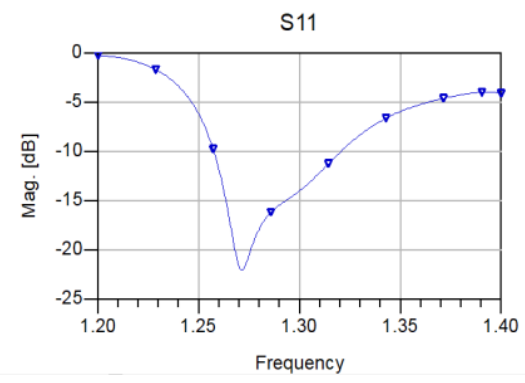

Figure 9. Simulation S-Parameters results versus frequency

\section{CONCLUSION}

The objectif of this paper is the validation of $\mathrm{CW}$ photonic transmitter using for generation of $\mathrm{THz}$ waves. As mentioned before the system is composed from photodetector, THz antenna, Low pass filter and DC probe. In the first step we have chosen the photodetector MSM-TPD. Then we pass to the optimization of the $\mathrm{THz}$ antenna which transmits the RF signal providing from the PD. After we move to the design of the low pass filter which blocks the RF signal to reach the DC probe Bias. And in the final step we have associated and simulated the whole system using an electromagnetic solver Momentum integrated in ADS until obtain the validate results. The final circuit is mounted on a multilayers GaAs substrate and having an area around $400.317 \times 167.16 \mu \mathrm{m}^{2}$.

\section{REFERENCES}

[1] Bertrand Gauvreau, " Ondes terahertz," Ecole Polytechnique de Montréal, 2500 chemin de Polytechnique, Montréal, QC H3T 1J4, Canada.

[2] J. Zbitou, C. Minot, X. Begaud, and B.Huyart, " Bow-tie Wideband Antenna Design for CW THz Photonic Transmitters," Progress In Electromagnetics Research Symposium, Cambridge, USA, July 2-6, 2008.

[3] S. Khireddine, M. Drissi, R. Soares," Flat Group Delay Low Pass Filters Using Two CPW Topologies, " * IETR, CNRS UMR 6164, INSA Rennes, France, *DAlightCom, Lannion, France.

[4] S. M. Sze, Kwok K. Ng" Physics of Semiconductor Devices" Third Edition A JOHN WILEY \& SONS, JNC., PUBLICATION.

[5] Vorgelegt von Diplom-Physiker Andreas Beling aus Bonn, "Periodic Travelling Wave Photodetectors with Serial and Parallel Optical Feed Based on InP."

[6] Shi, J.-W., K.-G. Gan, Y.-J. Chiu, Y.-H. Chen, C.-K. Sun, Y.-J. Yang, and J. E. Bowers, "Metal-semiconductormetal traveling-wave photodetectors," IEEE Photonics Technology Let-ters, Vol. 13, No. 6, 623-625, Jun. 2001.

[7] Chi-KuangSun, "THz Optoelectronics," UltraFastOptics Laboratory (UFO) Graduate Institute of Electro-Optical Engineering and Department of Electrical Engineering National Taiwan University Taipei, TAIWAN. 
[8] I.Moumane, J.Zbitou, A.Erkik. M.Latrach, O.Chakkor, A.Fouad "Design of CW THz Photonic transmitter based on Low Pass-Filter and Bow-tie Wideband Antenna" IJECE Vol 8, No 5:October part II 2018.

[9] Ming-Chun Tien, Hsu-Hao Chang, Ja-Yu Lu, Li-Jin Chen, Shih-Yuan Chen, Ruey-Beei Wu, Senior Member, IEEE,Wei-Sheng Liu, Jen-Inn Chyi, Senior Member, IEEE, and Chi-Kuang Sun, Senior Member, IEEE "Device Saturation Behavior of Submillimeter-Wave Membrane Photonic Transmitters".

[10] Yi-Chun Chen, An-Shyi Liu, Shih-Yuan Chen, Ruey-Beei Wu and Chi-Kuang Sun. "Design of Rampart Slot Array Antenna in Integrated $850 \mathrm{GHz}$ Photonic Transmitter “, Department of Electrical Engineering and Graduate Institute of Communication Engineering, Graduate Institute of Electro-Optical Engineering.

[11] I.Moumane, J.Zbitou, A.Erkik, Larbi El Abdellaoui, Abdelali Tajmouati,M.Latrach "Compact Photonic Transmitter Based on Annular Ring Antenna for THz Applications”, TELKOMNIKA Vol.16, No.5, October2018.

[12] James Sor Miniature "Low-Loss CPW Periodic Structures for Filter Applications", Student Member, IEEE, Yongxi Qian, Senior Member, IEEE, and Tatsuo Itoh, Fellow, IEEE

[13] Advanced Design System : https://www.keysight.com/.

\section{BIOGRAPHIES OF AUTHORS}

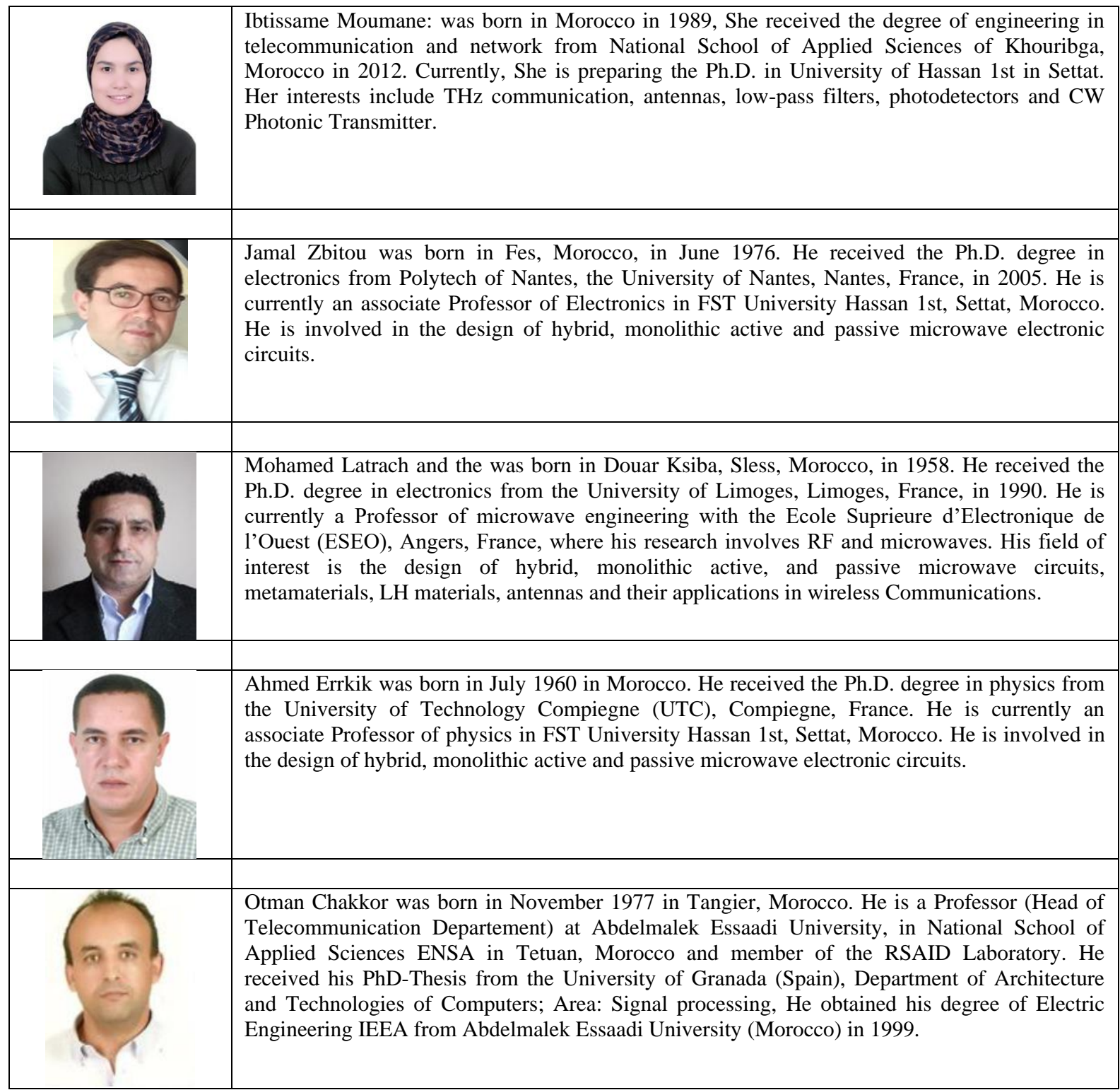

